\title{
Caracterización de lavas vítreas de fuentes y sitios arqueológicos del Formativo Temprano en la Subárea Circumpuneña: Resultados preliminares y proyecciones para la prehistoria atacameña
}

\author{
Andrea Seelenfreund H., ${ }^{1}$ Carole Sinclaire A., ${ }^{2}$ Patricio de Souza H., ${ }^{3}$ María Inés Dinator R., ${ }^{4}$
} Eugenia Fonseca P., ${ }^{5}$ Miguel Chesta A. ${ }^{6}$ y José Roberto Morales P. $^{7}$

\begin{abstract}
RESUMEN
Se entregan los resultados del estudio preliminar de caracterización por métodos fisicoquímicos (PIXE y EDXRF) y mineralógico de muestras líticas identificadas a nivel macroscópico como "basalto vítreo" procedentes de sitios arqueológicos de contextos del Período Formativo Temprano de la Subregión del río Salado, en el norte de Chile. Como resultado de los análisis petrog ráficos se constató que se estaba en presencia de dos tipos de roca, una andesita de prioxeno vítrea y una riodacita vítrea. Los análisis de caracterización permitieron establecer que los métodos usados pueden discriminar entre los distintos grupos químicos de rocas muestreadas. Los objetivos del trabajo se orientan a resolver preguntas de orden metodológico para el análisis de material lítico y a resolver por medio de la aplicación de estas técnicas problemas relativos a la circulación y producción de material lítico en un espacio y tiempo determinados de la prehistoria del norte de Chile.
\end{abstract}

Palabras claves: análisis de procedencia - andesitas vítreas - riodacitas vítreas - Subregión río Salado - Energy Dispersive X-Ray Fluorescence Analysis (EDXRF) Proton Induced X-Ray Emission (PIXE).

\begin{abstract}
Experimental and preliminary petrographic analysis and chemical characterization studies were carried out on lithic samples from archaeolog ical sites in the Salado subregion in northern Chile. Rock samples analyzed come from early Formative contexts and were performed on a rock
\end{abstract}

1 Escuela de Antropología, Universidad Academia de Humanismo Cristiano. Condell 343, Santiago, CHILE. Email: aseelenfreund@academia.cl

2 Museo Chileno de Arte Precolombino. Bandera 361, Santiago, CHILE. Email: csinclaire@museoprecolombino.cl

3 Universidad Católica del Norte. Correo San Pedro de Atacama, II Región, CHILE. Email: pdesouza@netexpress.cl

4 Departamento de Física, Facultad de Ciencias, Universidad de Chile. Las Palmeras 3425, Nuñoa, Santiago, CHILE. Email: mdinator@uchile.cl macroscopically identified as vitrified basalt. Results of the petrographic analysis indicated the presence of two types of rocks, a vitrified piroxite andesite and a vitrified rhyodacite. On the other hand chemical characterization showed that the methods used could successfully discriminate between different chemical groups. The objectives of the research were to test specific analytical techniques and to apply these results to answer question on the production and circulation of raw materials and lithic tools in the río Salado subregion in Northern Chile.

Key words: provenance analysis - vitrified andesites vitrified rhyodacites - Salado river Subregion - Energy Dispersive X-Ray Fluorescence Analysis - Proton Induced $X$-Ray Emission Analysis.

Recibido: abril 2004. Manuscrito revisado aceptado: septiembre 2004.

\section{Introducción}

El conocimiento arqueológico del Período Formativo ( $c a .1400$ AC-900 DC) en la Subregión del río Salado (Loa Superior, norte de Chile) ha avanzado sustancialmente en los últimos años, principalmente a partir de investigaciones que se han venido desarrollando por más de una década a través de tres proyectos FONDECYT consecutivos. ${ }^{8}$ Estos han abarcado temáticas como el arte rupestre, los sistemas de asentamiento y la producción lítica de las poblaciones que se desarrollaron durante ese período cultural (Gallardo et al. 1996 y 1999; Sinclaire et al. 1997; Rees 1998

5 Departamento de Laboratorio, SERNAGEOMIN. Tiltil 1993, Ñuñoa, Santiago, CHILE. Email: efonseca@sernageomin.cl

6 Departamento de Física, Facultad de Ciencias Universidad de Chile. Las Palmeras 3425, Ñuñoa, Santiago, CHILE. Email:mchesta@uchile.cl

7 Departamento de Física, Facultad de Ciencias, Universidad de Chile. Las Palmeras 3425, Ñuñoa, Santiago, CHILE. Email: rmorales@uchile.cl

8 Proyectos FONDECYT 1024-88, 1950102 y 1980200. 
Ms y 1999; Gallardo 2001; Gallardo 2004; Sinclaire 2004; Rees y De Souza 2004; De Souza 2004a).

Entre los resultados de estas investigaciones destaca el delineamiento de hipótesis que describen y caracterizan los sistemas de producción lítica en términos de la procuración, procesamiento y circulación de materias primas tanto locales como foráneas (Rees 1998 Ms y 1999; Rees y De Souza 2004; De Souza 2004a). En particular, sobre la base de análisis morfofuncionales y tecnológicos de los conjuntos líticos provenientes de los sitios formativos identificados en el área, se han definido dos sistemas productivos, asociados a las fases temprana (ca. 1400 AC-100 DC) y tardía (100900 DC) ${ }^{9}$ del Formativo (Sinclaire 2004). Ambos sistemas articularían de manera diferente su relación con zonas aledañas a través de su producción lítica y de sus redes de aprovisionamiento (Rees y De Souza 2004).

Durante el Formativo Temprano, la industria lítica de la población cazadora-recolectora y con incipiente pastoreo se caracteriza por el uso mayoritario de materias primas locales; presentarían una movilidad acotada a la localidad y con incursiones hacia la vecina puna ubicada en la actual frontera chileno-boliviana, en procura de recursos de caza y materias primas líticas. En cambio, durante la fase tardía del Formativo, que representa a poblaciones hortícolas y ganaderas, se establece una tendencia a utilizar materias primas provenientes de yacimientos más lejanos, de algún lugar al sur de la localidad de estudio (Salar de Atacama y puna adyacente) y del oeste (río Loa Medio y Alto Loa). Se desarrolla también de manera superlativa, durante esta fase tardía, la industria de cuentas de malaquita y crisocola cuyo volumen sugiere su orientación hacia el intercambio. Esta situación coincide con la apertura de la población local hacia zonas aledañas como el altiplano boliviano y el Noroeste Argentino, a través de mecanismos de intercambio, evidenciados por la gran cantidad de bienes foráneos, especialmente alfarería, que se registran en algunos sitios de esta zona (Castro et al. 1992; Sinclaire et al. 1997; Sinclaire 2004; Rees y De Souza 2004).

9 Este rango de fechas corresponde a la secuencia cronológica para el Período Formativo en la cuenca del río Salado, en base a fechas RC14 calibradas.
El sistema productivo lítico del Formativo Temprano se caracterizaría por un énfasis en la producción de piezas bifaciales grandes (particularmente puntas de dardo o de lanza) e instrumentos de uso doméstico como cuchillos, raederas y raspadores, la mayoría de los cuales presentan alto grado de formatización (De Souza 2004a; Rees y De Souza 2004). La gran mayoría de las materias primas utilizadas durante esta fase corresponderían supuestamente a rocas de origen local, si bien la utilización en menor grado de la obsidiana para la confección de puntas pequeñas sugiere la articulación del sistema basado, en algún grado, en relaciones de larga distancia para la adquisición de las materias primas.

Dentro de las materias primas más populares utilizadas para la confección de las puntas de formato grande se encuentra una en particular que presenta características macroscópicas distintivas, en especial su color gris muy oscuro y medianamente brillante, una textura fina de aspecto vidrioso, y la presencia continua de pequeñas inclusiones cristalinas a lo largo de sus superficies que le dan una apariencia "azucarada". Estas características le dan un aspecto como "entre y obsidiana". Es probable que en algunos otros contextos no analizados por nosotros haya sido confundida con alguna de estas dos materias primas. Anteriormente, en la literatura arqueológica regional se la denominaba "basalto" (Sinclaire 1985; Aldunate et al. 1986; Rees y De Souza 2004).

En la localidad de Linzor de la alta puna de la Subregión del río Salado fue descubierta la única cantera -y probable fuente de origen- de esta materia prima hasta ahora conocida por la arqueología regional. Se trata de un gran afloramiento que muestra señales de haber sido intensamente explotado. En el entorno hay una extensa área de depósito secundario de fragmentos de variado tamaño desprendidos por erosión desde el afloramiento, el que también evidencia signos de explotación. La cantera muestra esencialmente trabajo primario (desbaste de núcleos), con muy poca formatización de matrices. A la fecha, aún no se han realizado estudios sistemáticos de la cantera.

Desde una perspectiva cronológica, durante el Arcaico Temprano esta materia prima se usó con frecuencias moderadas a bajas. En el único sitio del Arcaico Medio detectado en la subregión (Alero Huiculunche), aparece con una frecuencia muy 
alta (De Souza 2004). Para el Arcaico Tardío, hasta ahora no poseemos sitios con dataciones o con contextos diagnósticos, por lo que no es posible hacerse una idea de su importancia. Para el Formativo Temprano se mantiene una alta frecuencia de uso de esta materia prima. Esto se evidencia, por ejemplo, en el sitio Los Morros-3, en las inmediaciones de la vega de Turi y en el Alero Toconce, del río homónimo. La alta frecuencia de esta materia prima en el sitio Los Morros-3 nos llevó a considerar que durante el Formativo Temprano habría existido una movilidad intensa hacia Linzor, en la alta puna. A la vez, se planteó la hipótesis de que los contactos hacia zonas aledañas se habrían dirigido principalmente hacia la zona del altiplano de Lipez, de la cual Linzor constituye su antesala geográfica. En el Formativo Tardío, en todos los sitios conocidos su frecuencia disminuye ostensiblemente a niveles mínimos o nulos (Rees y De Souza 2004).

La casi total ausencia de esta materia prima en los sitios del Formativo Tardío coincide con el término de la confección de aquellos artefactos para los cuales fue mayormente destinada, esto es, instrumentos bifaciales grandes, en su mayoría correspondientes a puntas de dardo o lanza. A la vez, esta situación del Formativo Tardío coincide con el aumento en la frecuencia de instrumentos en obsidiana y con el auge de la industria de microperforadores y cuentas, ambas situaciones que las vinculamos con la intensificación de las redes de intercambio y, a juzgar por los sitios por los que circula la obsidiana, con un cambio en el eje de relaciones con zonas aledañas, esta vez más dirigido hacia el Salar de Atacama y el Noroeste Argentino para la probable procura de esta materia prima (Lazzari 1998/1999; Rees y De Souza 2004).

Con el fin de contrastar y enriquecer los modelos de producción lítica anteriormente descritos, es de vital importancia determinar cuáles son efectivamente las fuentes de aprovisionamiento involucradas. En particular, es muy importante saber si la cantera en Linzor corresponde o no a la fuente utilizada por las poblaciones que, desde el Arcaico Medio hasta el Formativo Temprano, muestran en sus sitios arqueológicos la presencia de esta materia prima. En lo que respecta al Formativo Temprano, es necesario conocer si el asentamiento Los Morros-3 se proveyó o no de esta fuente para la confección de la mayoría de sus piezas bifaciales grandes. Evidentemente, y como se desprende de los modelos que hemos resumido, este es un problema cuyos alcances se encuentran mucho más allá de las puras consideraciones sobre las características organizacionales del sistema de producción lítica, involucrando a los sistemas de movilidad y asentamiento en su globalidad y a las vías de interacción con poblaciones vecinas. Hay que recordar que los temas de movilidad y de interacción han sido considerados como de vital importancia en la comprensión de la dinámica cultural de las poblaciones del Formativo Temprano en la Puna de Atacama (Núñez 1992 a y b, 1994, 1995; Núñez y Dillehay 1995).

De esta manera, como objetivo central de este trabajo se planteó primero identificar en términos geológicos la materia prima referida. En segundo lugar, conocer la composición fisicoquímica de ella y de esta manera poder correlacionar muestras seleccionadas de algunos sitios a la única cantera reconocida a la fecha y, finalmente, evaluar de manera preliminar si esta materia prima presente en los sitios arqueológicos de la subregión proviene de una o más fuentes de origen.

El trabajo fue realizado con un número limitado de muestras, exclusivamente de contextos arqueológicos del Formativo Temprano, incluyendo dos sitios que estando fuera de la subregión de estudio podrían hacer parte de los circuitos de circulación hipotéticamente planteados por Rees y De Souza (2004). También nos interesó evaluar la posibilidad de aplicar métodos analíticos nucleares (basados en el uso de haces de iones generados por aceleradores de partículas, o en neutrones generados en reactores nucleares) con infraestructura disponible en el país, a problemas de índole arqueológica y en especial su capacidad de discriminar otras materias primas que no sea obsidiana, lo que a la fecha no ha sido realizado en Chile y sólo de manera limitada en el extranjero (Newman y Nielsen 1987).

\section{Geología de la zona de estudio}

Los sitios arqueológicos de donde provienen las muestras seleccionadas se insertan en una macrorregión, de unos $240 \mathrm{~km}^{2}$, que integra la Subregión del río Salado, el vecino altiplano de Lipez del suroeste boliviano y el extremo norte de la cuenca del Salar de Atacama (localidad de Machuca) (Figura 1). 


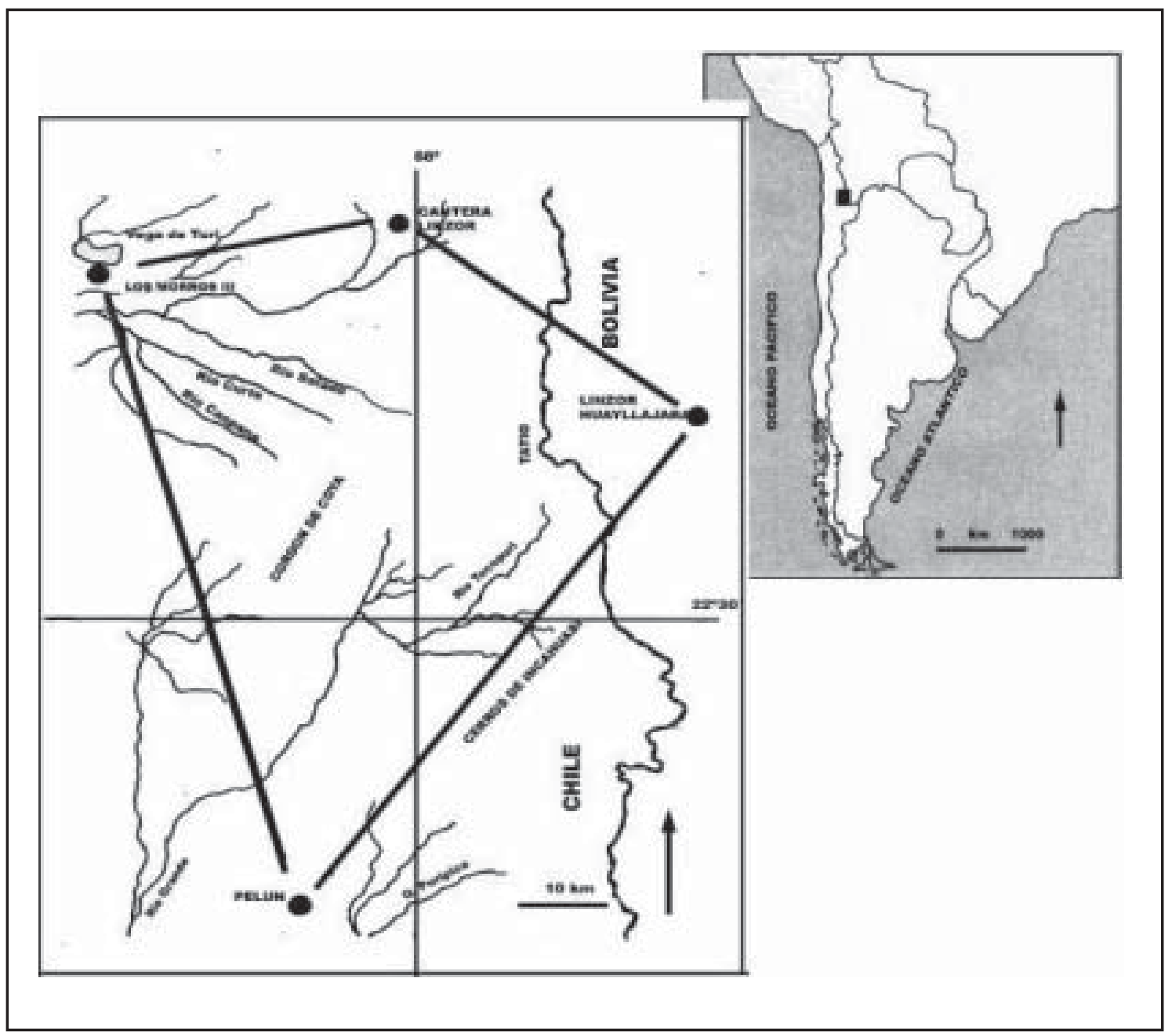

Figura 1. Mapa de ubicación de los sitios en la Subregión del río Salado y borde septentrional de la cuenca del Salar de Atacama.

La cuenca del río Salado se inserta dentro de una ecozona de quebradas (3000 a $3600 \mathrm{~m} . \mathrm{snm}$ ) y es el principal afluente del río Loa. Nace en la alta puna (Linzor) y en su recorrido va cortando el plateau volcánico con vegas y pastizales. A la altura de Ayquina, el río Salado corre por una profunda quebrada uniéndose al río Loa poco antes de la localidad de Chiu Chiu. Toda la Subregión del Salado está dominada por altos volcanes con erupciones de lavas andesíticas y dacíticas del Pleistoceno Tardío y Holoceno, que en la carta geológica de Chile lleva asociada la simbología Qv1 (Marinovic y Lahsen 1984).

El volcán Toconce, en cuyo faldeo sureste se sitúan la cantera y un taller prehispánico (Linzor), presenta andesitas de piroxeno en la parte alta de los flujos magmáticos y dacita/biotita en la parte baja de los mismos flujos de lava. Por otro lado, el cerro Chao, un domo volcánico ubicado al noroeste del volcán Toconce y al oeste del volcán León, presenta may ormente dacita/biotita. Hacia el sur -en dirección al Salar de Atacamay por unos $300 \mathrm{~km}$ en línea recta, esta cadena volcánica continúa con los volcanes Tocorpuri y Sairecabur, cuyas lavas también corresponden principalmente a andesitas de piroxeno y dacita/ biotita. Estos flujos magmáticos tienen principalmente su origen en las actividades volcánicas andesíticas que se inician en el Mioceno y continúan hasta hoy. Esta misma se encuentra depositada en forma discordante sobre un basamento del Mesozoico. 
Los volcanes de esta zona forman la parte más alta de la cordillera de los Andes, con alturas de más de $5000 \mathrm{~m}$ e integran el denominado grupo volcánico El Tatio, que consiste en estrato-volcanes de composición andesítica, a excepción del volcán Tocorpuri, que es un domo de lava riolítica. Las lavas andesíticas e ignimbritas son principalmente tobas dacíticas vítreas bien soldadas, atribuibles al Mioceno Medio y Superior. Movimientos tensionales posteriores asociados al levantamiento de los Andes habrían facilitado la efusión de material dacítico y tobas pumicitas soldadas, como, por ejemplo las ignimbritas riolíticas y sedimentos piroclásticos de los volcanes Linzor y Toconce, los cuales corresponden a la joven piroxita andesítica que fluyó del volcán Toconce (Davidson y Lahsen 1974).

\section{Material de estudio}

Las muestras analizadas provienen de cuatro sitios arqueológicos y de una cantera de aprovisionamiento de material lítico.

Los Morros-3 (Localidad de Turi, 3050 m.snm). Corresponde al asentamiento de mayor envergadura hasta ahora conocido para el Formativo Temprano, tratándose del sitio-tipo de la Fase I Los Morros (1400-500 AC) de la Subregión del río Salado (Sinclaire 2004). El sitio fue trabajado por Orellana y colaboradores (1969), de cuyas excavaciones se recuperó el material lítico estudiado, el cual proviene del estrato inicial de la ocupación de este asentamiento. De este mismo contexto se obtuvo un fechado radiocarbónico a partir de una muestra de carbón de fogón extraída por Orellana y colaboradores, de $2910 \pm 40 \mathrm{C}^{14} \mathrm{AP}$ (1250 a 990 AC Cal. BETA 149919). Se trata del único sitio de los seleccionados en esta investigación que cuenta con datación absoluta. El sitio, ubicado en un sector actualmente seco de la vega de Turi, está conformado por una veintena de recintos subcirculares semiaglutinados, con ocupaciones livianas y abundantes elementos de molienda en superficie.

Pelun (Localidad de Machuca, 3260 m.snm). El sector arqueológico de Pelun se ubica en el extremo septentrional del Salar de Atacama. Este sector fue recorrido en los años 50 por Le Paige (1958), quien recolectó numeroso material lítico de superficie e identificó sitios que en algunos casos atribuyó a lo que denominaba "transición al Mesolítico". Cinco décadas más tarde, en una breve expedición que algunos de nosotros realizamos al lugar, reconocimos una fuente secundaria de obsidiana, además de varios sitios (recintos habitacionales semiaglutinados, con molienda y talleres líticos), que preliminarmente adscribimos al Formativo Temprano atacameño, en base a la evidencia cerámica y lítica de superficie (De Souza et al. 2003). En al menos dos de estos sitios (a los que denominamos provisionalmente Pelun 1 y 3 ) fue detectada en superficie la presencia de la materia prima a la que hemos hecho referencia, en frecuencias bajas de instrumental bifacial similar al de Los Morros-3 (puntas de proyectil grandes, triangulares isósceles de base escotada). El reconocimiento de esta materia prima en los sitios de Pelun abre el interés sobre la posibilidad de identificar contactos de larga distancia entre las poblaciones del Salar de Atacama y aquellas de la cuenca del río Salado y río Loa, manifiestas a través de los materiales o los instrumentos líticos transportados.

Huayllajara-14 (Provincia de Lipez, Bolivia, 4340 m.snm). Este sitio, excavado por Axel Nielsen, (Instituto de Tilcara, Jujuy), corresponde a un refugio asociado a la explotación de recursos de avifauna adosado a un pequeño paredón en una quebrada de curso permanente que corre hacia la Laguna Colorada. Este presenta un nivel de ocupación cerámico (Formativo) y uno acerámico (Arcaico Tardío o posiblemente Formativo Temprano). De particular interés para nosotros es el segundo de ellos, que este investigador (Nielsen com. pers. 2003) atribuye a una población de cazadores recolectores y pastores tempranos que articulan circuitos de movilidad hacia la vecina Subregión del río Salado. En este nivel ocupacional se identificó macroscópicamente en su instrumental lítico, la misma materia prima motivo de este estudio, y de la cual una muestra fue facilitada por el Dr. Nielsen para este trabajo (Nielsen 2002).

Linzor (Localidad de Linzor, 4200 m.snm). Este sitio es la única fuente y cantera de extracción de esta materia prima conocida a la fecha. Corresponde a un afloramiento en los faldeos sureste del volcán Toconce, donde se observa mucho trabajo de desbaste primario, indicando que el material seleccionado fue trasladado a otros lugares 
para la confección de herramientas formatizadas. De hecho, a pocos kilómetros de distancia, a orillas de la vega de Linzor, encontramos un asentamiento con taller asociado. De esta cantera se obtuvieron algunas muestras al azar para su análisis.

\section{Descripción de las muestras}

Se analizó un total de siete muestras que provenían tanto de contextos arqueológicos y de la única fuente y cantera explotada registrada a la fecha. La procedencia y características de cada muestra se resumen en la Tabla 1. Las piezas se ilustran en la Figura 2.

\section{Metodología de análisis}

El análisis macroscópico de la lítica involucrada indicó que se trataba de una roca vítrea semejante a la obsidiana. Para identificar el tipo de roca se realizaron cortes delgados en los Laboratorios del Servicio Nacional de Geología y
Minería (SERNAGEOMIN), a las muestras $\mathrm{n}^{\mathrm{o}} \mathrm{s}$ 5, 6 y 7 observándose bajo el microscopio con luz cruzada y paralela. Esto permite la identificación mineralógica y adscripción de los materiales a las formaciones geológicas correspondientes.

Simultáneamente, cuatro muestras distintas de los mismos sitios fueron analizadas utilizando dos métodos físicos nucleares, en el Laboratorio de Física Nuclear de la Facultad de Ciencias de la Universidad de Chile: Proton Induced X-Ray Emission (PIXE) y Energy Dispersive X-Ray Fluorescence (EDXRF). El método PIXE consiste en ionizar átomos de la muestra mediante protones u otra partícula cargada. Los átomos ionizados al volver a su estado no ionizado emiten radiación electromagnética (fotones), la cual es característica de cada elemento. Los fotones emitidos son detectados y posteriormente procesados en unidades analizadoras, permitiendo identificar el átomo que los emitió (nivel cuantitativo). Análisis posteriores permiten determinar la

\begin{tabular}{|cllll|}
\hline $\mathbf{N}^{\circ}$ muestra & Muestra & \multicolumn{1}{c|}{ Procedencia } & \multicolumn{1}{c|}{ Características } & Análisis efectuado \\
\hline 1 & LM-3a & Los Morros-3 (B2, capa 2) & Lasca & PIXE y EDXRF \\
2 & Pe-3 & Pelun-3 (superficie) & Fragmento de punta de proyectil & PIXE y EDXRF \\
3 & Li & Huayllajara-14 (nivel inferior) & Lasca & PIXE y EDXRF \\
4 & LIN & Linzor (superficie) & Lasca de cantera & PIXE y EDXRF \\
5 & LINb & Linzor (superficie) & Lasca de cantera & CoXRF \\
6 & LM-3b & Los Morros-3 (B2, capa 2) & Trozo aberrante & EDXRF \\
& Pe1 & Pelun-1 (superficie) & Base de punta de proyectil & Corte petrográfico \\
7 & & Tabetrográfico \\
\hline
\end{tabular}

Tabla 1. Listados de muestras y análisis aplicado a cada una de ellas.

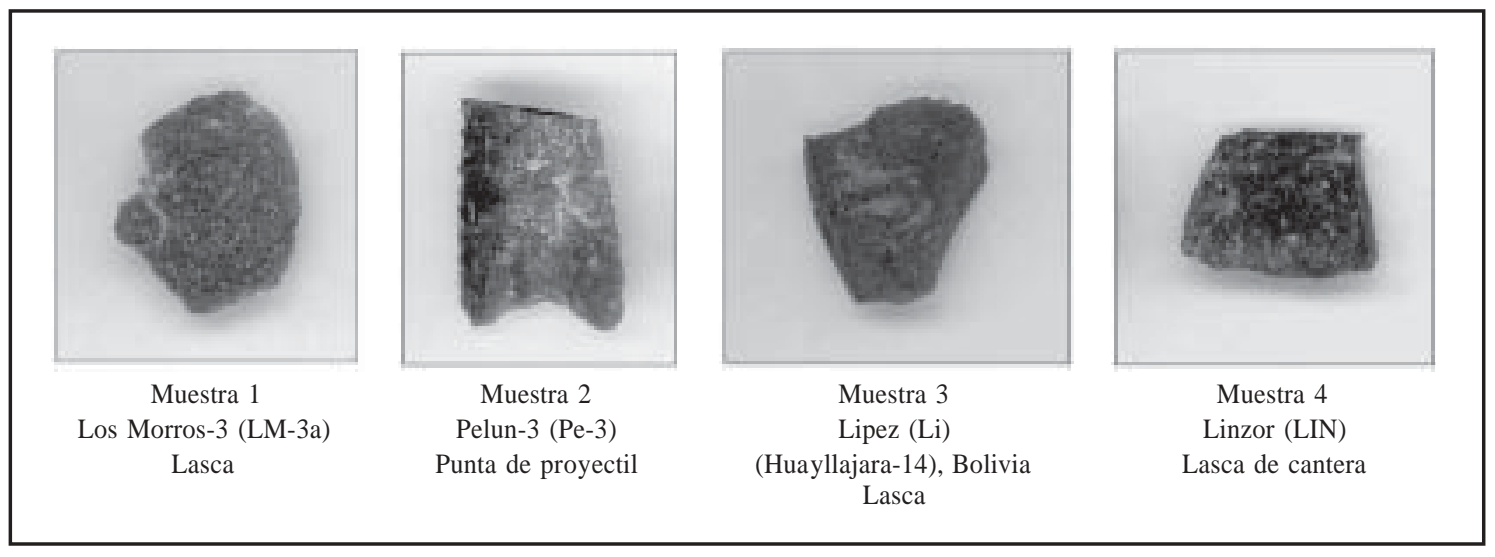

Figura 2. Las muestras analizadas en la primera serie de PIXE y EDXRF. 
concentración de elementos, tales como Al, $\mathrm{Si}, \mathrm{P}$, $\mathrm{S}, \mathrm{Ca}, \mathrm{Ti}, \mathrm{V}, \mathrm{Cr}, \mathrm{Mn}, \mathrm{Fe}$. Para la aplicación del método PIXE en este proyecto se usaron haces de partículas cargadas proporcionadas por el acelerador Van de Graaff de la Universidad de Chile, con energías desde 1.5 a $3.5 \mathrm{MeV}$. Dado que la probabilidad de ionizar el átomo es distinta para cada elemento y depende de la energía de las partículas cargadas incidentes, se ajusta la energía del haz para lograr la detección de los elementos más significativos en la muestra.

El método EDXRF consiste en ionizar los átomos de la muestra utilizando radiación electromagnética. Después de ionizados los átomos "no recuerdan" el agente responsable y, similarmente al método PIXE, emiten una radiación característica que permite realizar análisis cuantitativos y cualitativos de elementos. La aplicación del método EDXRF en este proyecto se realizó por medio de fuentes emisoras de fotones de $59.5 \mathrm{keV}$ y $22 \mathrm{keV}$, emitidos como resultado del decaimiento radiactivo de núcleos de ${ }^{241} \mathrm{Am} \mathrm{y}{ }^{109} \mathrm{Cd}$, respectivamente. $\mathrm{La}$ fuente de ${ }^{241} \mathrm{Am}$ permite obtener información sobre elementos como In, $\mathrm{Ba}, \mathrm{La}, \mathrm{Ce}, \mathrm{Sn}, \mathrm{Sb}$; en cambio, la fuente de ${ }^{109} \mathrm{Cd}$ es muy útil para elementos como $\mathrm{Co}, \mathrm{Ni}, \mathrm{Cu}, \mathrm{Zn}, \mathrm{As}, \mathrm{Zr}, \mathrm{Sr}, \mathrm{Rb}$, y $\mathrm{Br}$.

Los métodos anteriormente explicados pueden considerarse como complementarios en cuanto al conjunto de elementos que permiten analizar, ya que en el caso del PIXE la probabilidad de ionizar el átomo es decreciente con el número atómico de átomo (Z), en cambio, en el caso de las fuentes radiactivas dicha probabilidad es creciente con Z. Además, dado que en ambos casos las muestras emiten fotones dentro del mismo rango de energía, se utilizó el mismo equipo para la obtención de los espectros y su posterior análisis.

Las muestras $n^{\circ} \mathrm{s} 1,2,3$ y 4 fueron las primeras en someterse a análisis de PIXE y EDXRF. En primera instancia, éstas fueron analizadas como muestra entera y luego fueron reducidas a polvo a tamaño de grano del orden $10 \mu \mathrm{m}$, a fin de comparar resultados cualitativos y cuantitativos para ambos métodos. En una segunda instancia se analizaron con EDXRF las superficies de los cortes petrográficos de las muestras $n^{\circ} 5 y^{\circ} 6$. La muestra $n^{\circ} 7$ no fue sometida a EDXRF, por su reducido tamaño.

El sistema de adqusición de datos de PIXE y EDXRF consiste en una tarjeta ORTEC-TRUMP
(8K) emuladora de un mCA, instalada en un computador. A partir de las densidades y concentraciones elementales se realizó un análisis multifactorial de tipo cluster utilizando el código STATISTICA. En este caso se utilizó la modalidad de distancia euclideana. Los resultados de los análisis se indican en dendogramas para las muestras estudiadas.

\section{Análisis y resultados}

Los resultados del análisis de caracterización mineralógica por cortes petrográficos, sobre las muestras líticas $\mathrm{n}^{\circ} \mathrm{s} 5,6$ y 7, indicaron que nos encontramos frente a dos tipos de rocas, que visualmente no son distinguibles. Se trata de riodacitas vítreas y andesitas vítreas, ambas volcánicas muy comunes en la región, pero una intrusiva y la otra extrusiva, respectivamente (ver Tabla 2).

La riodacita vítrea se compone de $10 \%$ de fenocristales de plagioclasa (albita) y contiene escasos fenocristales de biotita. La masa fundamental es vítrea con textura de flujo y tiene indicios de ser de una erupción muy reciente. Sus cristales se encuentran orientados en la misma dirección que el flujo de la lava (ver Figura 3, muestra $\mathrm{n}^{\circ} 5$ ).

La andesita vítrea se compone de $50 \%$ de pequeños cristales de plagioclasas (andesinas) y piroxenos. En los intersticios se observa vidrio volcánico fresco de erupción reciente, posiblemente del Holoceno Temprano (ver Figura 3, muestras $\mathrm{n}^{\circ} 6 \mathrm{y} \mathrm{n}^{\circ} 7$ ).

Los resultados de los análisis de PIXE y EDXRF realizados a las muestras $n^{\circ} \mathrm{s} 1,2,3$ y 4 permitieron discriminar dos conjuntos diferentes en base a su composición química (ver Figura 4). Los espectros obtenidos muestran patrones de elementos semejantes (porque todas las muestras son rocas volcánicas), pero también diferencias. Al comparar estos resultados con aquellos de los

\begin{tabular}{|ll|}
\hline Muestra & Tipo de roca \\
\hline 5. LIN b & riodacita vítrea \\
6. LM-3b & andesita vítrea \\
7. Pe 1 & andesita vítrea \\
\hline
\end{tabular}

Tabla 2. Resultados de los cortes petrográficos. 

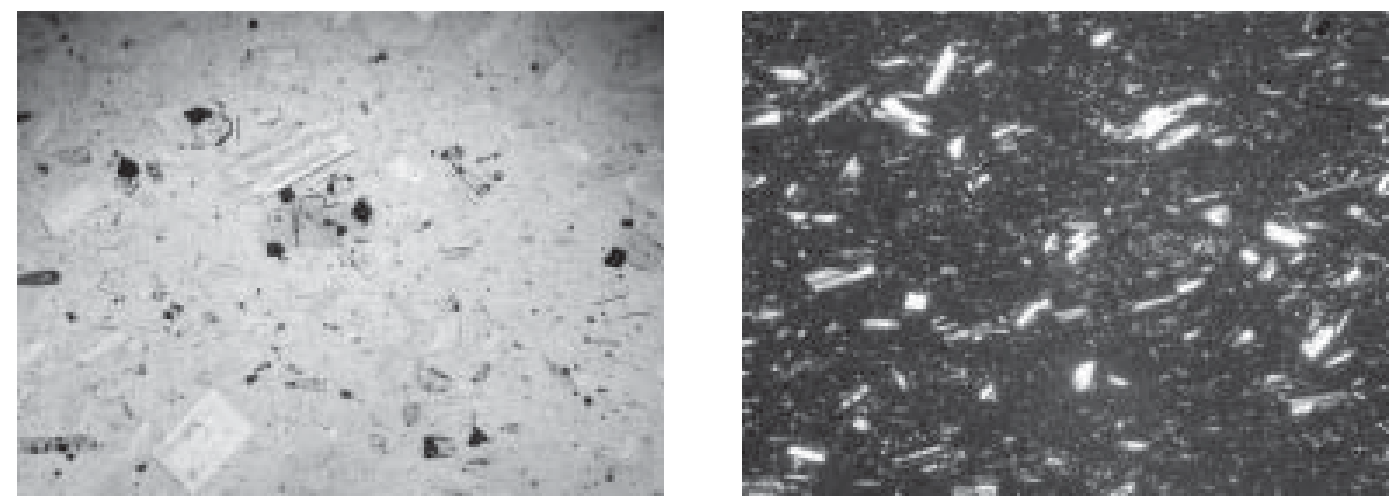

Muestra 5 Linzor cantera (LIN b)

Riodacita vítrea

Corte delgado bajo luz paralela y luz cruzada (aumento 40x)
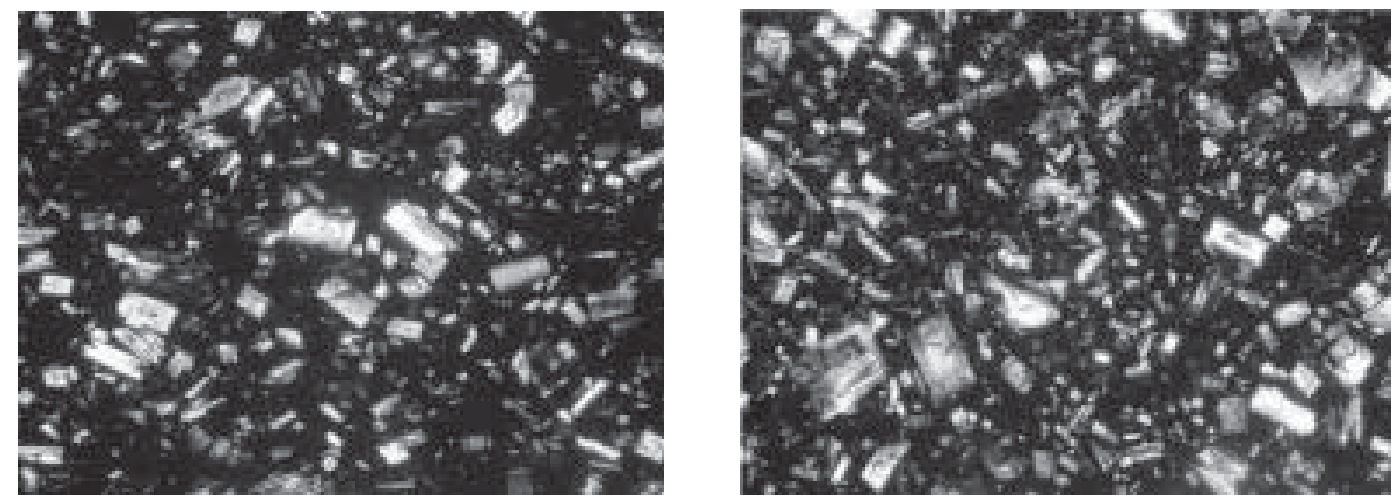

Muestra 6

Los Morros-3 (muestra LM-3b)

Andesita vítrea

Corte delgado bajo luz cruzada (aumento 40x)
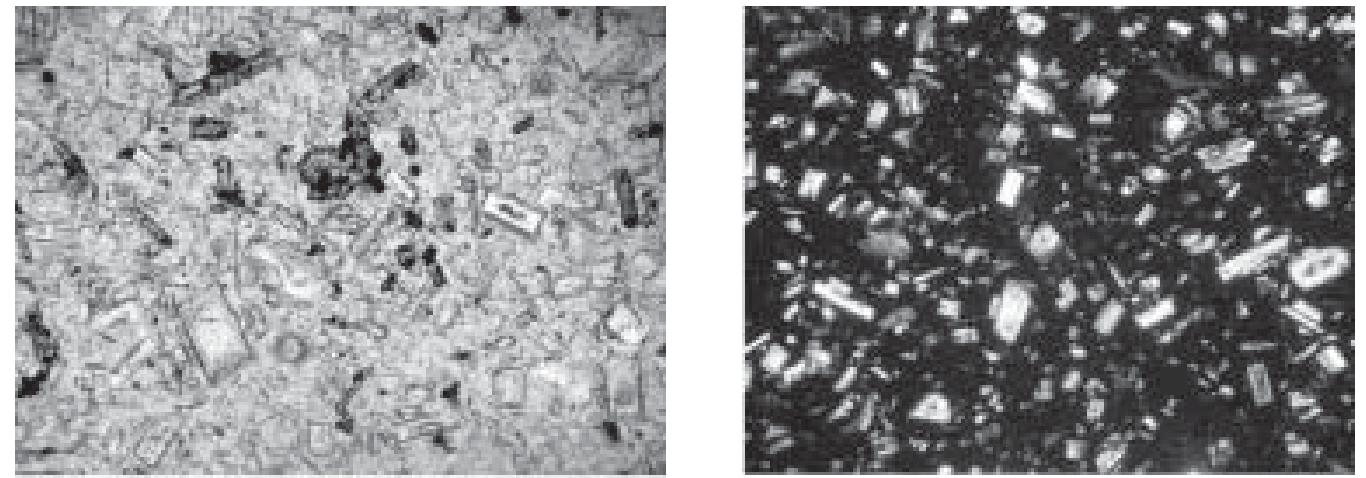

\section{Muestra 7}

Pelun-1 (Pe-1)

Andesita vítrea

Corte delgado bajo luz paralela y luz cruzada (aumento 40x)

Figura 3. Cortes mineralógicos de las muestras 5, 6 y 7. 


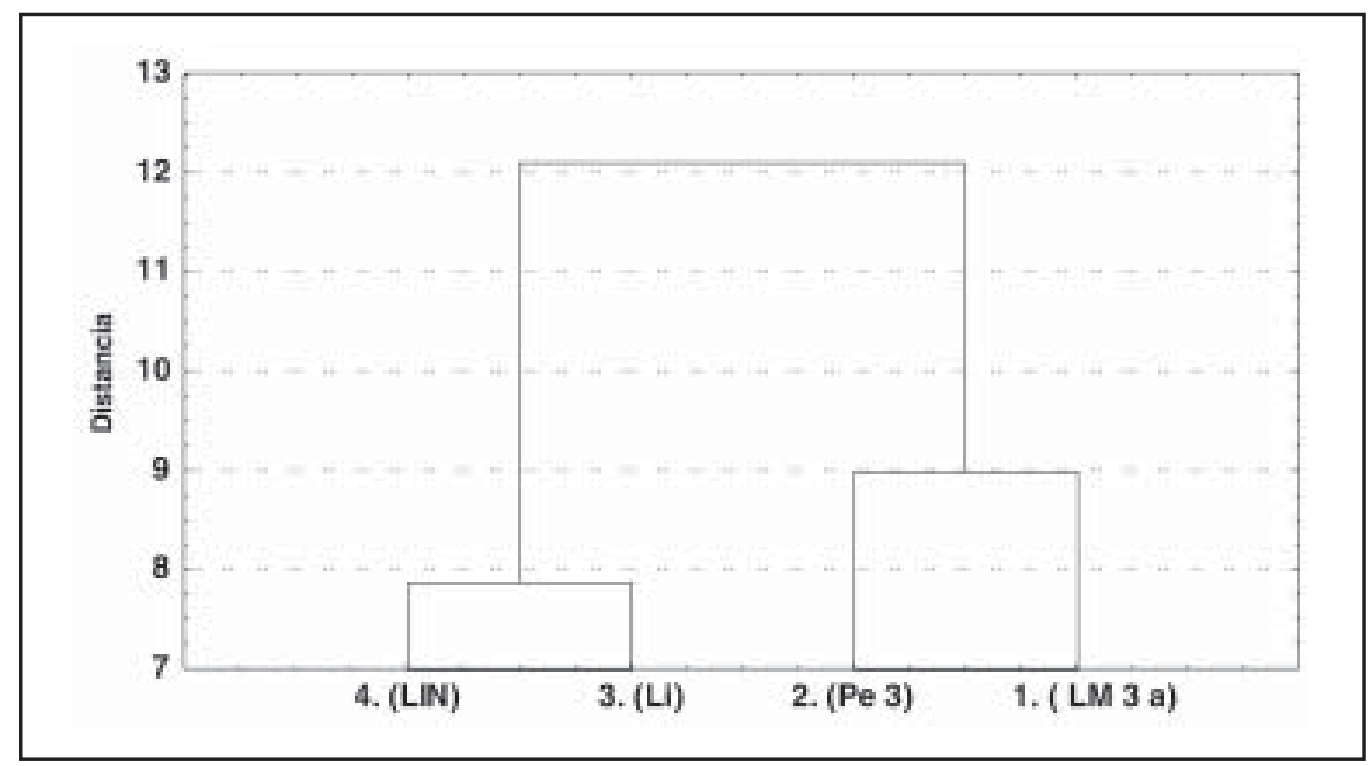

Figura 4. Dendograma de distancia euclideana para las muestras 1, 2, 3 y 4, considerando los espectros de PIXE y EDXRF.

cortes petrográficos sobre las otras muestras que suponíamos por sus características macroscópicas una misma roca, nos planteamos la posibilidad de que los resultados observados en los análisis fisicoquímicos tuvieran relación con esta diferencia observada en los cortes petrográficos. Para contrastar este supuesto, se procedió a analizar las muestras de las rocas sometidas a cortes petrográficos con EDXRF (muestras $\mathrm{n}^{\circ} 5 \mathrm{y} \mathrm{n}^{\circ} 6$ ).

Las concentraciones obtenidas de los elementos mayoritarios y trazas de la segunda instancia de análisis se integ raron con los primeros resultados y se presentan en la Tabla 3 .

En el dendograma de la Figura 5 se grafican los resultados de distancia euclideana para las seis muestras analizadas, comparando las concentraciones de 14 elementos para los elementos determinados por EDXRF.

\section{Discusión}

Los resultados del análisis de caracterización mineralógica y fisicoquímica indicaron que nos encontramos frente a tres grupos de rocas y no una, como habíamos pensado inicialmente de acuerdo a la observación macroscópica. Las dos muestras que provienen de Linzor $\left(\mathrm{n}^{\circ} 4 \mathrm{y} \mathrm{n}^{\circ} 5\right)$ junto a la de Lipez $\left(n^{\circ} 3\right)$ se agrupan en el cluster en un segmento distintivo, y por el corte delgado realizado a la muestra $\mathrm{n}^{\circ} 5$ podemos concluir que agrupa a riodacitas vítreas que son las que se encuentran en la cantera de Linzor. Otro grupo está conformado por las muestras de los sitios de Los Morros-3 y Pelun-3 ( $n^{\circ} 1$ y n $^{\circ} 2$ ), que muestran semejanzas entre sí. Sin embargo, sólo para una de las muestras se cuenta con corte petrográfico que la identifica como andesita vítrea. Un caso aparte lo plantea la otra muestra de Los Morros-3 $\left(n^{\circ} 6\right)$, que aun cuando sabemos por petrografía que corresponde a una andesita vítrea, a nivel de composición química no se correlaciona con ninguno de los dos grupos que identifica el cluster de la Figura 5.

Si bien los resultados obtenidos son sumamente preliminares por la condición experimental de este trabajo y el reducido tamaño de las muestras analizadas, podemos desprender de ellos algunas conclusiones tentativas y delinear hipótesis que permitirán orientar los próximos pasos a seguir en esta línea de investigación:

1) El sector de la cantera de Linzor que fue muestreado efectivamente presenta riodacitas vítreas según la petrografía de la muestra analizada. Esto no debe sorprender dado que en la geología regional se describen en la misma localidad formaciones de dacitas-biotitas e ignimbritas 


\begin{tabular}{|c|c|c|c|c|c|c|}
\hline \multicolumn{7}{|c|}{ Muestras y sitios asociados } \\
\hline \multirow[b]{2}{*}{ Elemento } & 1 & 2 & 3 & 4 & 5 & 6 \\
\hline & $\begin{array}{l}\text { Los Morros-3 } \\
\text { (LM-3a) }\end{array}$ & $\begin{array}{l}\text { Pelun-3 } \\
\text { (Pe-3) }\end{array}$ & $\begin{array}{c}\text { Lipez } \\
(\mathbf{L i})\end{array}$ & $\begin{array}{c}\text { Linzor } \\
(\text { LIN) }\end{array}$ & $\begin{array}{l}\text { Linzor } \\
(\text { LINb) }\end{array}$ & $\begin{array}{l}\text { Los Mor ros-3 } \\
\quad(\text { LM-3b) }\end{array}$ \\
\hline Al & 1.5 & 1.9 & 3.2 & 3.4 & & \\
\hline $\mathbf{S i}$ & 2.9 & 5.7 & 11.8 & 6.0 & & \\
\hline $\mathbf{K}$ & 0.39 & 0.60 & 1.02 & 0.55 & & \\
\hline $\mathrm{Ca}$ & 0.04 & 0.06 & 0.07 & B.L.D. & & \\
\hline $\mathbf{T i}$ & 0.05 & 0.06 & 0.06 & 0.09 & & \\
\hline Mn & N.O & N.O & B.L.D. & 0.066 & & \\
\hline $\mathrm{Fe}$ & 125.8 & 139.9 & 76.9 & 63.7 & 64.7 & B.L.D \\
\hline $\mathbf{N i}$ & 8.3 & N.O & N.O & N.O & N.O & N.O \\
\hline $\mathbf{R b}$ & 10.8 & 10.0 & 4.8 & 3.0 & 5.6 & 8.0 \\
\hline $\mathrm{Sr}$ & 9.3 & 10.1 & 4.9 & 4.1 & 3.8 & 5.7 \\
\hline $\mathrm{Zr}$ & 9.1 & 8.1 & 4.1 & 3.7 & 4.5 & 4.5 \\
\hline $\mathrm{Nb}$ & 1.23 & 0.73 & 0.10 & 0.30 & B.L.D & B.L.D \\
\hline $\mathbf{B a}$ & 45.7 & 44.9 & 40.0 & 35.2 & 47.4 & 20.1 \\
\hline $\mathrm{Ce}$ & 5.3 & 3.7 & B.L.D & B.L.D & 3.5 & 1.6 \\
\hline \multicolumn{7}{|c|}{$\begin{array}{l}\text { B.L.D. Elemento presente bajo el límite de detección } \\
\text { N.O. Elemento no observado }\end{array}$} \\
\hline
\end{tabular}

Tabla 3. Concentraciones elementales $\left(\mathrm{mg} / \mathrm{cm}^{3}\right)$.

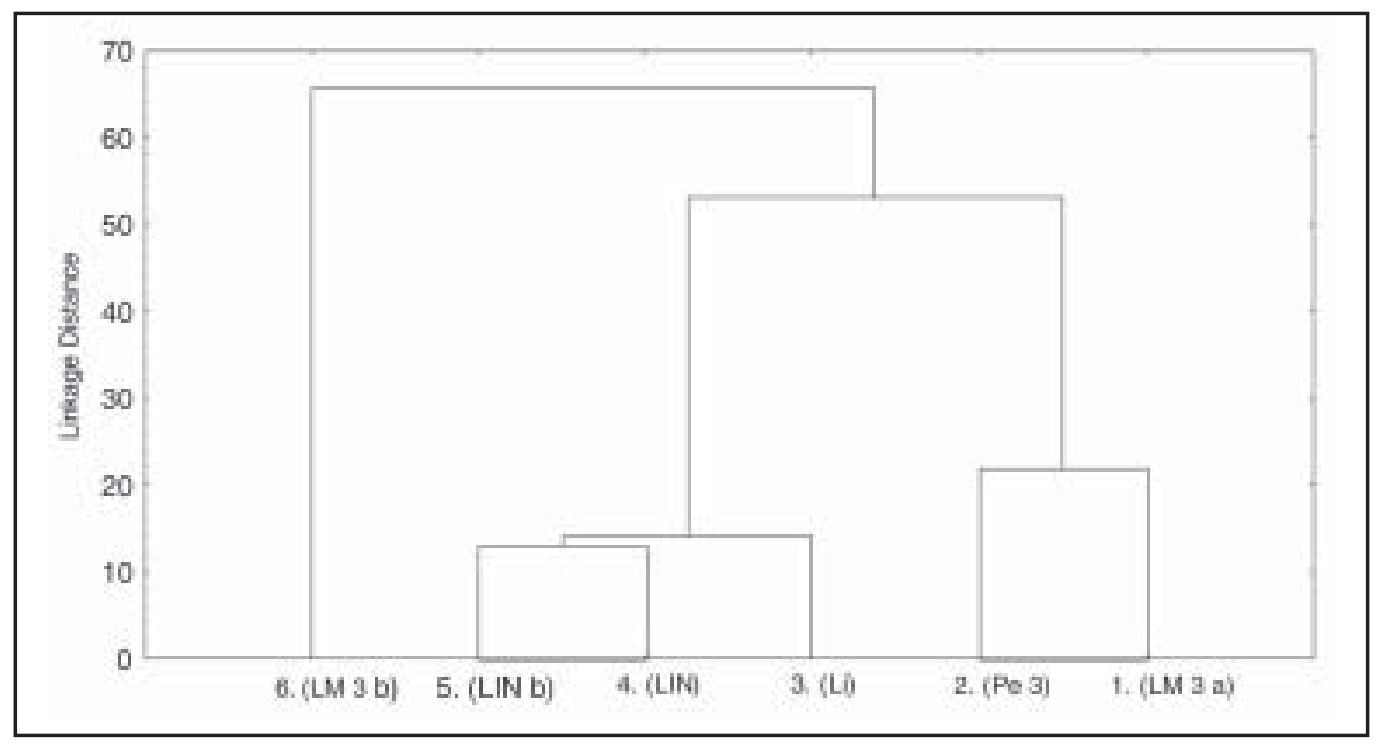

Figura 5. Dendograma de distancia euclideana considerando solamente los espectros de EDXRF para seis muestras.

riolíticas. En efecto, la riodacita vítrea identificada presenta fenocristales de biotita que son asociables a estas formaciones.

2) No conocemos la o las canteras de aprovisionamiento de andesitas vítreas identificadas en las muestras líticas de los sitios Los Morros-3 (Subregión del río Salado) y Pelun-3 (Localidad de Machuca). Esta situación no excluiría por completo la posibilidad de que en el sector donde se encuentra la cantera de Linzor existiera otra cantera de aprovisionamiento pero de andesitas, ya que la carta geológica nos indica la presencia de ambos tipos de roca. Esta hipótesis la sugiere la estrecha relación contextual observada entre el sitio Los Morros-3 y un taller lítico (Linzor-1), ubicado a corta distancia de la cantera, particularmente por cerámica compartida y porque ambos sitios formarían los extremos de una cadena operativa de la confección de las grandes puntas 
bifaciales (dardos y lanzas) características del Formativo Temprano en esta subregión (Rees y De Souza 2004). Por otro lado, la materia prima usada en Pelun-3 (muestra $\mathrm{n}^{\circ} 2$ ) se correlaciona químicamente con una de las materias primas analizadas del sitio Los Morros-3 (muestra $\mathrm{n}^{\circ} 1$ ), aun cuando no sabemos si esta última petrográficamente es una andesita vítrea. Los contextos arqueológicos y, particularmente, la industria de puntas de proyectil que comparten ambos sitios distantes unos $60 \mathrm{~km}$ en línea recta entre sí, podrían ser reflejo de vinculaciones culturales de sus poblaciones aun no comprendidas. Al usar materias primas similares, podríamos proponer que sus usuarios se proveían de la misma fuente: en el sector Linzor o en la zona puneña cercana a Pelun, como, por ejemplo, en las laderas del volcán Sairecabur, cerro Colorado o volcán Tocorpuri. Todas ellas presentan, según la carta geológica, andesitas de piroxeno semejantes a la descrita para el área de Linzor en el volcán Toconce.

3) La población de Los Morros-3 se está aprovisionando de andesitas vítreas de al menos dos fuentes distintas, según los resultados de los análisis fisicoquímicos. Esta observación podría tener asidero considerando la movilidad y el cierto oportunismo que caracterizaría a las poblaciones cazadoras-recolectores de este período para el aprovisionamiento de materias primas líticas. De ello da cuenta la relación con Pelun mencionada anteriormente y con el sector de Lipez en la alta puna boliviana, que se discute a continuación.

4) De los resultados de los análisis de PIXE y EDXRF se desprende una estrecha correlación entre las muestras de Lipez y Linzor (ver Figuras 4 y 5). Esta semejanza indica que la muestra de Lipez podría corresponder a riodacita, la misma de la cantera de Linzor, aunque todavía no está identificada petrográficamente. Esta situación detectada vendría a reforzar el planteamiento de Nielsen acerca de la estrecha relación que él encuentra entre los contextos formativos tempranos (especialmente lítica) del sitio Huayllajara con los que caracterizan a los asentamientos contemporáneos de la Subregión del río Salado. De hecho, se ha postulado que las poblaciones del Salado mantienen un sistema de asentamiento-subsistencia que integraría este sector del suroeste de la puna boliviana. En efecto, la gente de Lipez sería la misma que se traslada desde el Salado ha- cia la alta puna en pos de recursos de avifauna lacustre y obsidianas (A. Nielsen, com. pers. 2003; Sinclaire 2004) y la cantera de Linzor se ubica en la ruta natural de acceso a esta última zona.

\section{Conclusiones}

Los resultados obtenidos a través de los análisis fisicoquímicos PIXE y EDXRF permiten concluir que estos métodos son apropiados para distinguir las diferencias en la composición elemental de este tipo de rocas. Sin embargo, una muestra estadísticamente relevante podrá reducir la variabilidad observada en ellas. Los resultados actuales pueden diferenciar tres grupos de materias primas, uno que incluye material de la fuente de Linzor con el sitio de Huayllajara, en Lipez, otro que reúne la muestra del sitio de Pelun-3 con una de las muestras del sitio Los Morros-3 y un último grupo que corresponde a la segunda muestra de Los Morros-3.

Al iniciar este estudio, no sospechábamos que el denominado "basalto vítreo" en realidad eran dos rocas distintas, una andesita y la otra riodacita. Esto claramente nos plantea la necesidad de enfrentar futuros estudios con una metodología que contemple en sus primeros pasos el análisis mineralógico-petrográfico de cada una de las muestras que se analicen posteriormente por vía fisicoquímica. Hay que recordar que en la literatura especializada a la fecha sólo existen escasas experiencias con otras materias primas volcánicas no obsidianas, como andesitas, riodacitas y basaltos, las que implican problemas y procedimientos de análisis diferentes al ámbito de las obsidianas, entre ellos, que existe la posibilidad de que en un mismo flujo (o fuente) se encuentren variaciones mineralógicas por distintos procesos de formación y enfriamiento de las lavas. Esto puede llevar a que en una misma formación lávica existan tanto riodacitas como andesitas. Como ejemplo, podemos mencionar los trabajos de Newman y Nielsen (1987) que por medio de análisis EDXRF pudieron discriminar entre distintas fuentes de riodacitas, originalmente clasificadas como andesitas vitrificadas, de una misma zona geológica en el suroeste de los Estados Unidos. Los elementos traza que se pueden discriminar en la composición de las rocas por medio de EDXRF son los adecuados para distinguir entre fuentes de estas características, así como lo de- 
muestran nuestros resultados a pesar de lo limitado de la muestra utilizada en este trabajo exploratorio.

Las similitudes entre el sitio de Lipez y la cantera de Linzor son de sumo interés puesto que verifican la conexión que sospechábamos entre la alta puna adyacente al Salado y el altiplano de Lipez. Además, es importante la conexión vista entre los sitios Pelun y Los Morros, los que se distancian entre sí por unos $60 \mathrm{~km}$. Esta situación sugiere que durante el Formativo Temprano esta materia prima se usó a lo largo de un espacio tan amplio como el que separa la localidad de Turi (Los Morros-3) con la de Pelun, en lo que podrían constituir rutas de tráfico y/o movilidad de poblaciones entre la cuenca del Loa y la del Salar de Atacama. De esta forma, se hace necesario evaluar la idea planteada anteriormente respecto a la forma o modalidad que asumen los contactos interregionales durante esta época, tanto hacia el altiplano de Lipez como hacia el Salar de Atacama. Por lo menos, en lo que respecta al aprovisionamiento de materiales líticos éste no sería tan local como se ha postulado (Rees y De Souza 2004).

Por otra parte, dado que los modelos de flujo de materiales líticos que hemos propuesto para el Formativo Temprano plantean como un punto de gran importancia la conexión Linzor-Los Morros, nos ha llamado particularmente la atención que los materiales líticos provenientes de estos sitios no se muestren agrupados. Al respecto, las posibilidades son: 1) que se trata de un problema del tamaño de la muestra que sobredimensiona las diferencias entre éstas; esto mismo podría estar afectando las diferencias mineralógicas vistas a nivel de corte delgado, ya que la fuente de Linzor es muy extensa y presenta según la carta geológica tanto andesitas como riodacitas, y 2) el modelo que conecta Los Morros con la alta puna adyacente a través de la fuente de Linzor es incorrecto, siendo necesaria su reformulación.

Por último, los resultados indican acceso a materiales líticos que visualmente son idénticos, pero que provienen posiblemente de fuentes distintas, como es el caso de las dos muestras de Los Morros-3 que no resultan agrupadas.

Independiente de las consideraciones anteriores, se hace claro que en la medida en que análisis como el presentado puedan hacerse más rutinarios y puedan contar con muestreos de mejor cobertura estadística, se podrá avanzar notoriamente en la comprensión de los mecanismos de movilidad y de interacción en el espacio circumpuneño.

Agradecimientos Estamos comprometidos con Francisco Gallardo, Charles Rees y el Dr. Axel Nielsen, quienes facilitaron las muestras para este estudio. Agradecemos también la colaboración de Felipe Llona (SERNAGEOMIN) por la molienda de las muestras. El apoyo de la Universidad de Chile fue fundamental en proporcionar el Acelerador Van de Graaff y el Laboratorio de Análisis de Física. Agradecemos a SERNAGEOMIN por el uso de su infraestructura. Este trabajo es consecuencia de una investigación iniciada bajo el Proyecto FONDECYT 1980200.

\section{REFERENCIAS CITADAS}

ALDUNATE, C., J. BERENGUER, V. CASTRO, L. CORNEJO, J. L. MARTINEZ y C. SINCLAIRE. 1986. Cronología y asentamiento en la región del Loa Superior. DIB-Universidad de Chile, Santiago.

CASTRO, V., C. ALDUNATE, J. BERENGUER, L. CORNEJO, C. SINCLAIRE y V. VARELA, 1992. Relaciones entre el Noroeste Argentino y el norte de Chile: El sitio 02-Tu-002, vegas de Turi. En Taller de costa a selva: Producción e intercambio entre los pueblos agroalfareros de los Andes Centro-Sur, M. E. Albeck (Ed.), pp. 215239. Instituto Interdisciplinario de Tilcara/Universidad de Buenos Aires, Jujuy.
DE SOUZA, P. 2004. Cazadores recolectores del Arcaico Temprano y Medio en la cuenca superior del río Loa: Sitios, conjuntos líticos y sistemas de asentamiento. Estudios Atacameños 27: 7-44.

2004a. Tecnologías de proyectil durante los períodos Arcaico y Formativo en el Loa Superior: Una aproximación inicial a partir de las puntas líticas. Actas del XV Congreso Nacional de Arqueología Chilena, pp. 61-76. Arica.

DE SOUZA, P., C. SINCLAIRE, R. MOLINA y F. GALLARDO, 2002. Una nota sobre el hallazgo de una fuente secundaria de obsidiana en la quebrada de Pelun (Locali- 
dad de Machuca, San Pedro de Atacama). Boletín de la Sociedad Chilena de Arqueología 33/34: 81-83.

DAVIDSON, J. y A. LAHSEN, 1974. Antofagasta-El TatioLaco, Guide Book, Excursión A-2. En International Symposium on Volcanology: Andean and Antarctic volcanology problems. J. Davidson y A. Lahsen (Eds.), pp. 1-61. Santiago.

GALLARDO, F., 2001. Arte rupestre y emplazamiento durante el Formativo Temprano en la cuenca del río Salado (Desierto de Atacama, norte de Chile). Boletín del Museo Chileno de Arte Precolombino 8: 83-97.

2004. El arte rupestre como ideología: Un ensayo acerca de pinturas y grabados en la localidad del río Salado (Desierto de Atacama, norte de Chile). Actas del XV Congreso Nacional de Arqueología Chilena, pp. 427-440. Arica.

GALLARDO, F., F. VILCHES, L. CORNEJO y C. REES, 1996. Sobre un estilo de arte rupestre en la cuenca del río Salado (norte de Chile). Chungara 28 (1-2): 353-354.

GALLARDO, F., C. SINCLAIRE y C. SILVA, 1999. Arte rupestre, emplazamiento y paisaje en la cordillera del Desierto de Atacama. En Arte rupestre en los Andes de Capricornio, J. Berenguer y F. Gallardo (Eds.), pp. 57-96. Museo Chileno de Arte Precolombino, Santiago.

LAZZARI, M., 1998/1999. Nuevos datos acerca de la procedencia de obsidianas de sitios de la falda occidental del Aconquija y áreas aledañas. Cuadernos del Instituto $\mathrm{Na}$ cional de Antropología y Pensamiento Latinoamericano 18: $243-256$.

LE PAIGE, G., 1958. El precerámico en la cordillera atacameña y los cementerios del Período Agroalfarero de San Pedro de Atacama. Anales de la Universidad del Norte 3.

MARINOVIC, N. y A. LAHSEN, 1984. Carta Geológica de Chile $N^{\circ}$ 58: Hoja Calama, Región de Antofagasta. Servicio Nacional de Geología y Minería, Santiago.

NEWMAN, J. R. y R. NIELSEN, 1987. Inicial notes on the $\mathrm{X}$-Ray fluorescence characterization of the rhyodacite sources of the Taos Plateau, New Mexico. Archaeometry 29 (2): 262-274.

NIELSEN, A., 2002. Contribución al estudio del tráfico circumpuneño durante el Período Formativo. Revista Arqueología 12. En prensa.

NUÑEZ, L., 1992 a. Cultura y conflicto en los oasis de San Pedro de Atacama. Editorial Universitaria, Santiago.

-1992 b. Ocupación arcaica en la Puna de Atacama: Secuencia, movilidad y cambio. En Prehistoria Sudameri- cana: Nuevas perspectivas, B. Meggers (Ed.), pp. 283-307. Taraxacum, Washington D. C.

1994. Emergencia de complejidad y arquitectura jerarquizada en la Puna de Atacama: Las evidencias del sitio Tulan-54. En Taller de costa a selva: Producción e intercambio entre los pueblos agroalfareros de los Andes Centro-Sur, M. E. Albeck (Ed.), pp. 85-115. Instituto Interdisciplinario de Tilcara/Universidad de Buenos Aires, Jujuy.

_ 1995. Evolución de la ocupación y organización del espacio atacameño. En Agua, ocupación del espacio y economía en la región atacameña: Aspectos dinámicos, P. Pourrut y L. Núñez (Eds.), pp. 18-60. Orstom y Universidad Católica del Norte, Antofagasta.

NUÑEZ, L. y T. DILLEHAY, 1995. Movilidad giratoria, armonía social y desarrollo en los Andes Meridionales: Patrones de tráfico e interacción económica. Universidad Católica del Norte, Antofagasta.

ORELlANA, M., C. URREJOLA y C. THOMAS, 1969. Nuevas investigaciones en el río Salado (informe preliminar). Actas del $V$ Congreso Nacional de Arqueología Chilena, pp. 113-127. La Serena.

REES, C., 1998 Ms. Materiales líticos tallados de contextos alfareros tempranos de la Subregión del río Salado, norte de Chile. Manuscrito en poder del autor.

-1999. Elaboración, distribución y consumo de cuentas de malaquita y crisocola durante el Período Formativo en la vega de Turi y sus inmediaciones, Subregión del río Salado, norte de Chile. En Los tres reinos: Prácticas de recolección en el Cono Sur de América, C. Aschero, M. A. Korstanje y P. M. Vuoto (Eds.), pp. 41-57. Instituto de Arqueología y Museo de Antropología, Universidad Nacional de Tucumán, Tucumán.

REES, C. y P. DE SOUZA, 2004. Producción lítica durante el Período Formativo en la Subregión del río Salado. Actas del XV Congreso Nacional de Arqueología Chilena, pp. 453-465, Arica.

SINCLAIRE, C., 1985. Dos fechas radiocarbónicas del alero Chulqui, río Toconce. Noticia y comentario. Chungara 14: 71-79.

2004. Prehistoria del Período Formativo (ca. 1000 AC800 DC) en la cuenca del Alto Salado, región del Loa Superior: Un estado de la cuestión. Actas del XV Congreso Nacional de Arqueología Chilena, pp. 619-639. Arica.

SINCLAIRE, C., M. URIBE, P. AYALA y J. GONZALEZ, 1997. La alfarería del Período Formativo en la Región del Loa Superior: Sistematización y tipología. Contribución Arqueológ ica 5: 285-314. 